\title{
49. ON THE SIZES OF COMETARY NUCLEI
}

\author{
V. P. KONOPLEVA and L. M. SHUL'MAN \\ Main Astronomical Observatory, Ukrainian Academy of Sciences, Kiev, U.S.S.R.
}

\begin{abstract}
Three methods have been developed for determining the sizes of cometary nuclei. They are all based on the spotted model for an icy nucleus. The first method follows from the relationship between the icy (unshielded) area and the total absolute brightness, although the quantity determined is actually a function of the radius and the shielding coefficient. The second method is based on analysis of the secular brightness decrease and the assumption that it is possible to establish when a new surface layer of dust starts to form; in practice, this method is found to be applicable only in the cases of $\mathrm{P} /$ Pons-Winnecke and $\mathrm{P} /$ Tuttle. The third method is also based on the secular brightness decrease, and it requires some assumption about the volume fraction of dust in the nucleus.
\end{abstract}

The total gas and dust production of an icy comet depends mainly on the size of the nucleus. There are few data on the radii of cometary nuclei, and these are inconsistent, as is clear from the estimates given in Table $I$ for the radius of $P /$ Halley at its 1910 return.

TABLE I

Estimates of the radius of the nucleus of $\mathbf{P} /$ Halley

\begin{tabular}{llll}
\hline$R_{\max }(\mathrm{km})$ & $R_{\min }(\mathrm{km})$ & $R(\mathrm{~km})$ & Author \\
\hline- & - & 0.75 & Sekanina (1960) \\
- & - & 1.0 & Orlov (1945, 1960) \\
- & 1.0 & - & Dobrovol'skij (1953) \\
- & - & 2.0 & Sekanina (1962) \\
- & - & 20 & Vorontsov-Vel'yaminov (1946) \\
- & 250 & - & Dubyago (1950) \\
450 & - & - & Bouska and Vanýsek (1967) \\
- & $3.1-14.6$ & - & Whipple (1963) \\
\hline
\end{tabular}

a This estimate refers to the 'original' $\mathrm{P} /$ Halley.

Many different methods have been used for the determination of nuclear radii. These have been based on the absolute brightness of the nucleus, the intensity of the continuous spectrum, dynamical properties, and so on. In addition to the references cited in Table I we mention here Vorontsov-Vel'yaminov (1945), Kostyakova (1966), Baldet (1951), Bobrovnikoff (1951), Hasegawa (1968), Houziaux (1959), Mianes et al. (1960), Richter (1958, 1963), Roemer (1966), and Whipple (1951). However, total absolute brightness has been used for this purpose for only a few comets (Dobrovol'skij, 1952; Sekanina, 1960). Since absolute magnitudes are known for practically all comets, it is of interest to use this vast material to obtain estimates of the total emission area, and for periodic comets the radius of the nucleus as well.

It is assumed that the surface of a nucleus consists of exposed regions of ice, with spots of mineral crust (spotted model). Since the temperature of a nucleus and the gas 
density at the surface do not depend on the radius, there is a universal relationship between the area of emission of parent-molecules and the total absolute brightness. The universality of the relationship is violated only by the variety of chemical composition, and then only slightly.

The total number of $\mathrm{C}_{2}$ molecules may be obtained in two ways:

(1) from the mass conservation law

$$
\mathrm{N}_{\mathrm{C}_{2}}=4 \pi R^{2} n_{01} v_{01} \tau_{1} \alpha(1-\xi),
$$

where $n_{01}$ and $v_{01}$ are the density and velocity of gas at the nuclear surface, and $\tau_{1}$ is the lifetime of $\mathrm{C}_{2}$ molecules, these quantities being referred to unit heliocentric distance; $R$ is the radius of the nucleus, $\alpha$ is a geometric factor, and $\xi$ is the fraction of the surface screened by mineral crust (the shielding coefficient).

(2) from the total visual magnitude $H$. If $f$ is the oscillator strength of the Swan system, then

$$
f N_{C_{2}}=10^{32.2-0.4 H} \text {. }
$$

From Equations (1) and (2) it follows that

$$
R \sqrt{f(1-\xi)}=\frac{10^{11.1-0.2 H}}{\sqrt{4 \pi \alpha n_{01} v_{01} \tau_{1}}} \mathrm{~km}
$$

where the quantities in the denominator on the right-hand side are in c.g.s. units. This relation gives us the first method (henceforth referred to as method I) for estimating the radius of a nucleus.

Assuming $f=0.003$ (Golden, 1967) and $4 \pi \alpha n_{01} v_{01} \tau_{1}=10^{22} \mathrm{~mol} \mathrm{~cm}^{-2}$, we have calculated values of $R \sqrt{ }(1-\xi)$ for each apparition of all the periodic and nonperiodic comets observed up until 1968. With a few exceptions the values for the short-period comets decrease with time.

The question arises as to whether the brightness decrease is due to the shrinking of the nucleus (thawing) or the growth of the shielding coefficient $\xi$ (screening). We have already discussed the evolution of nuclei (Shul'man, 1972) and have shown that the quantity $1-\xi$ decreases with time by the law

$$
\begin{aligned}
\log (1-\xi)= & 0.53 \frac{R \delta_{d}}{C_{D} v_{o p}} \frac{f_{v}}{1-f_{v}} \frac{1-a_{\mathrm{eq}} / a_{\max }}{\log a_{\mathrm{max}} / a_{\min }} \\
& \times\left[\int_{0}^{2 \pi}(1+e \cos \theta)^{n-2} \mathrm{~d} \theta\right] \frac{q^{3 / 2}}{(1+e)^{n-1.5}} v_{t r}
\end{aligned}
$$

where $C_{D} \approx 10$ is the drag coefficient of the grain, $f_{v}$ is the volume fraction of dust in the nucleus, $e$ and $q$ are the usual orbital elements, $\theta$ is the true anomaly, $n$ the photometric parameter, $\nu_{t r}$ the number of revolutions since the comet's origin, $\delta_{d} \approx 1$ is the mass density of the grain, $a_{\max }$ and $a_{\min }$ are the extreme radii of grains, $a_{\mathrm{eq}}$ is the radius of a grain in equilibrium at the nuclear surface, and $v_{o p} \approx 10^{4} \mathrm{~cm} \mathrm{~s}^{-1}$ is the gas velocity at the surface at perihelion. 
Equation (4) is valid for $\xi \leqslant 0.9$; otherwise the coefficient 0.53 must be replaced by 3.5 , because after $\xi$ has reached the value 0.9 a second layer of dusty crust starts to form, and the evolution proceeds more rapidly.

If observations exist of a comet both before and after the formation of the second layer, we have another method for determining the nuclear radius (method II). We obtain at two points $\nu_{1}$ and $\nu_{2}$ (measured according to the number of revolutions $\nu$ ) in the first part of the absolute brightness curve the function, following from Equation (3),

$$
\psi(v)=\log (R \sqrt{1-\xi)}=0.1-0.2 H
$$

and form

$$
\nabla_{1}=\frac{\psi\left(\nu_{2}\right)-\psi\left(\nu_{1}\right)}{\nu_{2}-\nu_{1}}=-0.2 \frac{H\left(\nu_{2}\right)-H\left(\nu_{1}\right)}{\nu_{2}-\nu_{1}}
$$

The situation is illustrated in Figure 1 , the variation of $\psi$ with $\nu$ during this stage being denoted by the straight line $A B$. We do the same for two points $\nu_{1}^{\prime}$ and $\nu_{2}^{\prime}$ in the

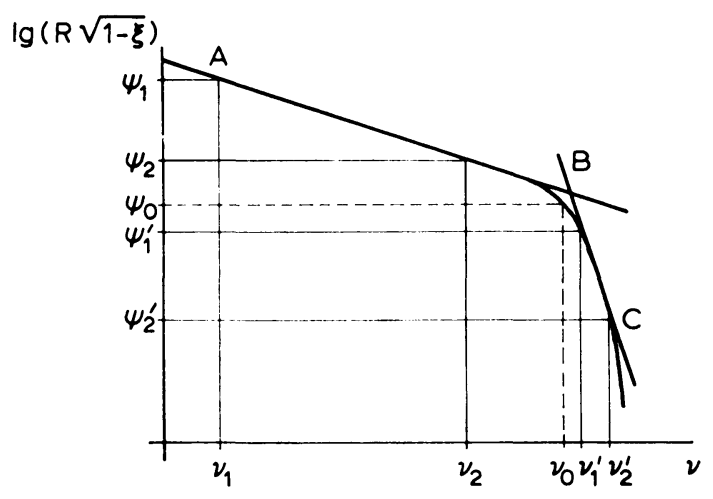

Fig. 1. Variation of $\log (R \sqrt{ }(1-\xi))$ with time.

second part of the brightness curve, this stage being denoted by the line $B C$. This gives us a possibility of testing the validity of the spotted model. If the model is correct, we must have that $\nabla_{1}(A B): \nabla_{1}(B C) \approx 0.15$. We can locate the point $B$ (the number of revolutions from discovery then being denoted by $\nu_{0}$ ) and derive the radius of the nucleus from Equation (3), taking $\xi=0.9$. Thus,

$$
R=10^{\psi(B)+0.5}
$$

Equation (4) can be written in the form

$$
\log (1-\xi)=\nabla_{2} R \frac{f_{v}}{1-f_{v}} \nu_{t r}
$$

Assuming that $a_{\mathrm{eq}} \ll a_{\max }$ and $\log \left(a_{\max } / a_{\min }\right) \approx 7$, we can easily calculate the $\nabla_{2}$ coefficient for each comet. If screening is the main reason for the secular decrease of 
brightness, we can neglect the variation in radius. It follows from Equations (5), (6), and (8) that

$$
\nabla_{1} \simeq \frac{1}{2} \nabla_{2} R \frac{f_{v}}{1-f_{v}}
$$

which enables us to calculate $f_{l}$. Finally, by evaluating Equation (8) at the point B we can obtain

$$
v_{t r}=\left[\frac{1-f_{v}}{f_{v} R\left|\nabla_{2}\right|}\right]
$$

the square brackets indicating the integral part, and the true age of the comet follows by multiplying $\nu_{t r}$ by the revolution period. There is another possibility of checking the theory, for it is obvious that we must have $v_{t r}(B) \geqslant \nu_{0}$.

In practice we are generally not able to locate the point $B$, and method II turns out to be applicable only to the two short-period comets Pons-Winnecke and Tuttle: see Figure 2 and Table II, where $\nu_{o b}$ gives the total number of revolutions made by the comet since discovery.

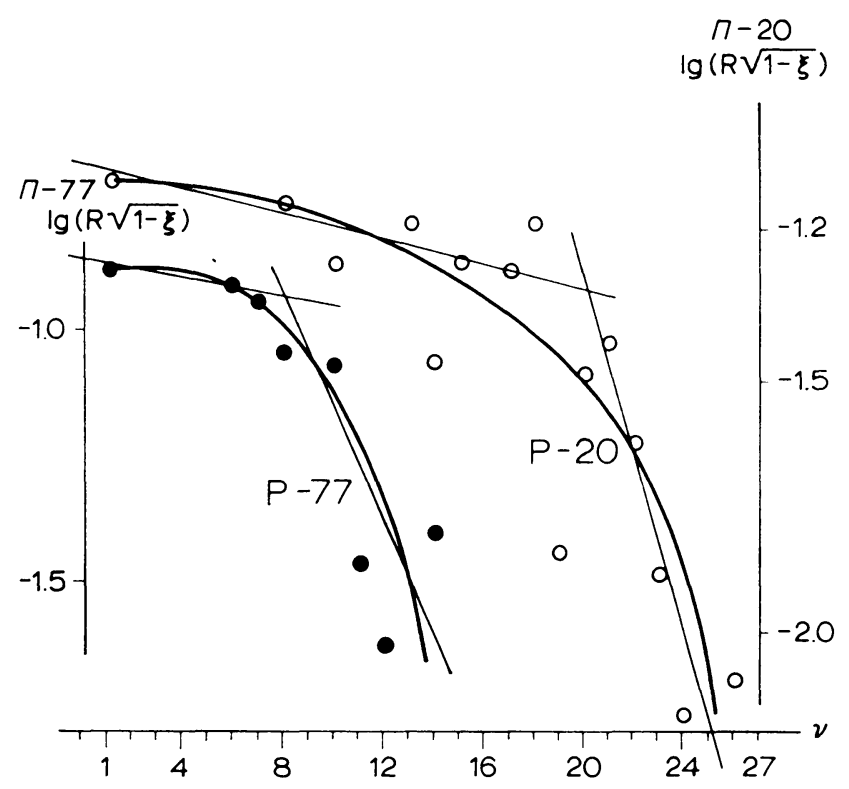

Fig. 2. Variation of $\log (R \sqrt{ }(1-\xi))$ with time for $\mathrm{P} / \mathrm{Pons}-$ Winnecke $(\mathrm{P}-20$; points denoted by $\mathrm{O})$ and $\mathrm{P} /$ Tuttle (P-77; point denoted by $\bullet)$.

For a particular comet we have no real way of knowing whether it is in the process of forming its first layer (the $A B$ region), its second layer (the $B C$ region), or even some subsequent layer, although there is a high probability of observing it in the first stage. In general we obtain the product $R \sqrt{ }(1-\xi)$ only, or using Equation (9), the product 
TABLE II

Nuclear radii of $\mathrm{P} /$ Pons-Winnecke and $\mathrm{P} /$ Tuttle

\begin{tabular}{lrlllll}
\hline Comet & $\nu_{0}$ & $f_{v}$ & $R(\mathrm{~km})$ & $\nu_{o b}$ & $\nu_{t r}$ & Origin \\
\hline Pons-Winnecke & 20 & 0.34 & 0.40 & 27 & 28 & $1814 \pm 30$ \\
Tuttle & 8 & 0.09 & 1.35 & 14 & 31 & $1554 \pm 100$ \\
\hline
\end{tabular}

Earlier estimates for P/Pons-Winnecke are: $0.6-3.5 \mathrm{~km}$ (Roemer, 1966), $0.2 \mathrm{~km}$ (Baldet, 1951), 4-20 km (Bouška and Vanỷsek, 1967; Richter, 1963), 82 km (Whipple, 1951).

$R f_{v} /\left(1-f_{v}\right)$. In order to estimate the radius we must make some supposition about the dust content $f_{v}$ (method III).

Values of $\nabla_{1}$ have been obtained from the preliminary smoothed curves of absolute brightness for several periodic comets. A number of comets, e.g., Halley, GriggSkjellerup, Encke, and Brooks 2, have periodic variations in absolute brightness. Some comets, e.g., Grigg-Skjellerup, Perrine-Mrkos, and Holmes (Hasegawa, 1968), must have been discovered during outbursts, if the strong decrease in the absolute brightness after the first apparition is real; this question requires additional treatment, and in a number of cases we have excluded first apparition data from our analysis.

Estimates of the radii are given in Table III for most of the comets having $v_{o b} \geq 10$. Here, $N$ is the actual number of apparitions, $\nu_{i}-\nu_{k}$ gives the revolution numbers of the

TABLE III

Derived nuclear radii for periodic comets with $v_{o b} \geqslant 10$

\begin{tabular}{lrrrrrrrr}
\hline Comet & $N$ & $\nu_{o b}$ & $\nu_{i}-v_{k}$ & $R_{i} \sqrt{ }(1-\xi)$ & $R_{k} \sqrt{ }(1-\xi)$ & $R(0.1)$ & $\hat{R}(0.1)$ & $R(0.3)$ \\
\hline Encke & 48 & 56 & $11-56$ & 0.46 & 0.08 & 11.37 & 0.54 & 2.95 \\
& & & $13-53$ & 0.35 & 0.12 & 7.70 & 0.37 & 2.00 \\
Grigg-Skjellerup & 11 & 14 & $5-14$ & 0.06 & 0.02 & 6.37 & 0.30 & 1.65 \\
Tempel 2 & 14 & 19 & $1-19$ & 0.30 & 0.11 & 1.46 & 0.07 & 0.38 \\
& & & $5-19$ & 0.24 & 0.11 & 1.42 & 0.07 & 0.37 \\
de Vico-Swift & 3 & 21 & $1-21$ & 0.57 & 0.02 & 4.13 & 0.20 & 1.07 \\
Tempel 1 & 4 & 17 & $1-3$ & 0.41 & 0.16 & 8.32 & 0.40 & 2.16 \\
& & & $1-17$ & 0.35 & 0.03 & 3.00 & 0.14 & 0.78 \\
Tuttle-G.-K. & 4 & 20 & $1-20$ & 0.10 & 0.04 & 1.53 & 0.07 & 0.40 \\
d'Arrest & 11 & 18 & $4-18$ & 0.31 & 0.07 & 3.11 & 0.15 & 0.81 \\
Perrine-Mrkos & 5 & 12 & $3-12$ & 0.05 & 0.01 & 5.26 & 0.25 & 1.36 \\
Kopff & 9 & 10 & $1-10$ & 0.42 & 0.07 & 3.98 & 0.19 & 1.03 \\
Finlay & 8 & 13 & $1-13$ & 0.22 & 0.06 & 5.56 & 0.26 & 1.44 \\
Biela & 6 & 13 & $6-13$ & 0.55 & 0.44 & 2.07 & 0.10 & 0.54 \\
Wolf & 11 & 12 & $1-11$ & 1.05 & 0.03 & 4.50 & 0.21 & 1.17 \\
Holmes & 4 & 11 & $2-11$ & 0.24 & 0.04 & 2.27 & 0.11 & 0.59 \\
Borrelly & 8 & 10 & $1-10$ & 0.29 & 0.05 & 5.43 & 0.26 & 1.41 \\
Brooks 2 & 10 & 11 & $1-11$ & 0.66 & 0.02 & 5.36 & 0.26 & 1.30 \\
Faye & 15 & 17 & $4-17$ & 0.91 & 0.06 & 4.23 & 0.20 & 1.10 \\
Crommelin & 6 & 19 & $9-19$ & 0.83 & 0.10 & & & \\
Halley & 32 & 32 & $19-32$ & 7.56 & 1.99 & 13.72 & 0.65 & 3.56 \\
\hline
\end{tabular}


range over which the absolute brightness curve was smoothed, $R(0.1)$ and $R(0.3)$ are nuclear radii (in $\mathrm{km}$ ) obtained by method III (the assumed value of $f_{v}$ being given in the parentheses), and $\hat{R}(0.1)$ is the effective radius on the assumption that the comet is in its second stage of evolution.

The results obtained here can be used to forecast the absolute brightness of periodic comets at their next apparitions. For example, the absolute magnitude of $\mathrm{P} / \mathrm{Halley}$ is expected to be about 5.6 in 1986.

\section{References}

Baldet, F.: 1951, Astronomie 65, 289.

Bobrovnikoff, N. T.: 1951, in J. A. Hynek (ed.), Astrophysics, New York, Toronto and London, p. 302.

Bouška, J. and Vanýsek, V.: 1967, Fyzika Komet, Prague.

Dobrovol'skij, O. V.: 1952, Byull. Stalinabad-Dushanbe Astron. Obs. No. 6.

Dobrovol'skij, O. V.: 1953, Byull. Stalinabad-Dushanbe Astron. Obs. No. 8, 3.

Dubyago, A. D.: 1950, Astron. Zh. $27,5$.

Golden, S. A.: 1967, J. Quant. Spectr. Radiative Transfer 7, 225.

Hasegawa, I.: 1968, Mem. Kyoto Univ. Ser. Phys. Astrophys. Geophys. Chem. 32, 37.

Houziaux, L.: 1959, Acad. Roy. Belg. Bull. Cl. Sci. 45, 218.

Kostyakova, E. B.: 1966, Problemy Kometnoj Fotometrii Inf. Byull. No. 10, 65.

Mianes, P., Grudzinska, S., and Stawikowski, A.: 1960, Ann. Astrophys. 23, 788.

Orlov, S. V.: 1945, Golova Komety i Klassifikatsiya Kometnykh Form, Leningrad.

Orlov, S. V.: 1960, Priroda Komet, Moscow.

Richter, N.: 1958, Sterne 34, 11, 215.

Richter, N.: 1963, The Nature of Comets, Methuen, London.

Roemer, E.: 1966, Mem. Acad. Roy. Sci. Liège Ser. 5 12, 23.

Sekanina, Z.: 1960, Publ. Astron. Inst. Charles Univ. Ser. II No. 28.

Sekanina, Z.: 1962, Publ. Astron. Inst. Charles Univ. Ser. II No. 36A.

Shul'man, L. M.: 1972, this Symposium, p. 271.

Vorontsov-Vel'yaminov, B. A.: 1945, Astron. Zh. 22, 317.

Vorontsov-Vel'yaminov, B. A.: 1946, Astrophys. J. 104, 226.

Whipple, F. L.: 1951, Astrophys. J. 113, 464.

Whipple, F. L.: 1963, in The Moon, Meteorites and Comets, Vol. IV of the series: The Solar System (ed. by B. M. Middlehurst and G. P. Kuiper), University of Chicago Press, Chicago and London, p. 639. 